\title{
PERSPECTIVAS DE LA ECONOMIA PESQUERA ANDALUZA ANTE EL INGRESO DE ESPAÑA EN LA C.E.E. (Análisis de las relaciones con terceros países)
}

José Luis OSUNA LLANEZA*

\section{INTRODUCCION}

Hablar de la integración de España en la C.E.E. es hablar de futuro y vincular el hecho a las repercusiones que tendría en la economía pesquera andaluza de cara a sus pesquerías en terceros países supone una limitación - marcada por ese factor tiempo ya citado- que imposibilita pronunciamientos contundentes y análisis cerrados; pues, el devenir de los años puede deparar situaciones nuevas que hoy no alcanzamos a ver:

En el intento de cumplir el objetivo del artículo, nuestra exposición va a tener dos partes claramente diferenciadas. Una primera, en la que trataremos de describir las pesquerías andaluzas fuera de sus aguas atendiendo a su localización, su significación económica (en base a los parámetros estructurales básicos: flota, empleo y producción) y un breve análisis histórico de los acuerdos pesqueros que la afectan.

En la segunda parte, abordaremos la cuestión central que da título a este trabajo y analizaremos, - primero, de forma general, más tarde, particularizando por zonas de faenaje- aquellos aspectos tanto positivos como negativos que plantea la integración de España en el Mercado Común Europeo al sector pesquero andaluz.

Finalmente, a modo de epílogo, y con intención de contrastar el análisis reflexionaremos brevemente en torno a la posibilidad contraria; es decir, sobre qué sucedería si España no se integrase en la C.E.E. 
RELACION DE EMBARCACIONES DE PESCA ANDALUZAS QUE FAENAN EN EL CALADERO SAILARIANO-MARROQUI, CON ESPECIFICACION DE SU TRB, HP Y NUMERO DE TRIPULANTES, SEGUN SUS DIFERENTES PUERTOS BASE

\begin{tabular}{|c|c|c|c|c|c|c|c|c|c|c|c|c|c|c|c|c|}
\hline & \multicolumn{4}{|c|}{ Número de Embarcaciones } & \multicolumn{4}{|c|}{ Tonelaje Registro Bruto } & \multicolumn{4}{|c|}{ Caballos de Vapor } & \multicolumn{4}{|c|}{ Tripulantes } \\
\hline & Arrastre & Cerco & $\begin{array}{c}\text { Artes } \\
\text { Menores }\end{array}$ & Total & Arrastre & Cerco & $\begin{array}{c}\text { Artes } \\
\text { Menores }\end{array}$ & Total & Arrastre & Cerco & $\begin{array}{c}\text { Artes } \\
\text { Menores }\end{array}$ & Total & Afrastre & Cerco & $\begin{array}{c}\text { Artes } \\
\text { Menores }\end{array}$ & Total \\
\hline Isla Cristina & 26 & - & - & 26 & 2.077 .19 & - & - & 2.077 & 7.940 & - & - & 7.940 & 233 & - & - & 233 \\
\hline Lepe & 9 & - & - & 9 & 693,03 & - & - & 693.03 & 3.103 & & - & 3.103 & 86 & $\cdot$ & - & 86 \\
\hline Punta Umbria & 49 & - & - & 49 & $4.196,24$ & - & - & $4.196,24$ & 14.225 & - & - & 14.225 & 436 & - & - & 436 \\
\hline Huelva & 34 & - & - & 34 & 4476,23 & - & - & 4.476 .23 & 12.701 & - & - & 12.701 & 408 & - & - & 408 \\
\hline Sanlucar & 8 & - & - & 8 & 521,72 & - & - & 521,72 & 2.134 & - & - & 2.134 & 81 & - & - & 81 \\
\hline Puerto de Sta Maria & 80 & - & - & 80 & 7.428 & - & - & 7.428 & 21.336 & - & - & 21.336 & 940 & - & - & 940 \\
\hline Cádiz & 7 & - & - & 7 & 1.478 & - & - & 1.478 & 4.565 & - & - & 4.565 & 97 & & - & 97 \\
\hline Barbate & - & 47 & 2 & 49 & - & 2.960 .32 & 57,29 & $3.017,61$ & - & 18.970 & 320 & 19.290 & - & 1.371 & 19 & 1390 \\
\hline Tarifa & - & 4 & 19 & 23 & - & 59.49 & 435,88 & 495,37 & - & 277 & 1.798 & 2.025 & & 27 & 114 & 141 \\
\hline Algeciras & 47 & 2 & 59 & 108 & 4.673 & 183 & 3.087 & 7.943 & 16.793 & 566 & 10.351 & 27.710 & 607 & 39 & 602 & 1248 \\
\hline Malaga & 8 & - & - & 8 & 491 & - & - & 491 & 1.730 & - & & 1.730 & 78 & - & - & 78 \\
\hline Motnl & 14 & - & 3 & 17 & 696,33 & - & 60,86 & 757,19 & 3076 & - & 488 & 3.564 & 126 & - & 16 & 142 \\
\hline Adra & - & 25 & - & 25 & - & $1.436,08$ & - & $1.436,08$ & - & 9244 & - & 9.244 & - & 663 & & 663 \\
\hline Almería & 48 & 8 & - & 56 & $2.903,2$ & 525,33 & - & $3.328,54$ & 13.202 & 3.046 & - & 16.248 & 512 & 146 & - & 657 \\
\hline Total Andalucia & 330 & 86 & 83 & 499 & $29.633,99$ & $5.064,22$ & 3641,03 & $38.339,19$ & 100.805 & 32.053 & 12.957 & 145.815 & 3.604 & 2.246 & 751 & 6.601 \\
\hline
\end{tabular}

Fuente Flaboración propia en base a los datos facilitados por las Cofradias de Pescadores respectivas y correspondientes al primer cuatrimestre de 1983 


\section{DESCRIPCION DE LAS PESQUERIAS ANDALUZAS EN AGUAS DE OTROS PAISES.}

\subsection{Significación económica por zonas de faenaje.}

La dependencia de la economía pesquera andaluza de caladeros cuyas aguas no le pertenecen viene dada, fundamentalmente, por el hecho de que dos tercios de su producción pesquera procede de caladeros situados en terceros países y principalmente de los que a continuación vamos a destacar.

\subsubsection{Costa sahariano-marroquí.}

Existen dos zonas claramente diferenciadas dentro de estas costas y vienen delimitadas por el Cabo Num, al Sur y al Norte del mismo.

El caladero del Sur de Cabo Num es pobre en marisco (dedicación primordial dentro de la estructura pesquera andaluza) pero, muy ricos en cefalópodos y, por tanto, presenta gran interés y expectativas para la flota congeladora. No sólo "per se", sino como alternativo en situaciones de dificultades en otros bancos pesqueros - hecho comprobado cuando surgieron problemas con Mauritania y que permitió que muchas unidades de esta zona pasasen a faenar mas al Norte, en el caladero a que estamos haciendo referencia.

El caladero del Norte de Cabo Num es un auténtico "cajón de sastre" para la flota de Andalucía ya que en el mismo tienen cabida distintos tipos de flota-desde la embarcación con 35 Tn. hasta la de 400-y sus especies se prestan a todos los tipos de artes habitualmente utilizados en el Sur-desde el arrastre y el cerco, hasta las llamadas artes menores, palangre, votanta...-.

El de las costas sahariano-marroquís es pues, un caladero a la medida del sector pesquero andaluz - que se atribuye su descubrimiento-- y del cual depende hasta tal punto que no resulta exagerado decir que buena parte de la flota pesquera del Sur fue construida en función de ese caladero y sus especies.

Según datos de las Cofradías de Pescadores Andaluzas, cerca de quinientos barcos de nuestra región faenan en ese caladero --aunque no todos ejercen la actividad durante todo el año de forma permanente- lo que supone el $19 \%$ del total de la flota pesquera. Esta flota desplaza un TRB de unas $38.400 \mathrm{Tn}$. y tiene una potencia instalada en sus motores de 145.815 caballos de vapor; todo ello viene a suponer, aproximadamente, el $24 \%$ y el $25 \%$ del total del tonelaje y la potencia de la flota pesquera andaluza. 
Para ver la importancia de la actividad extractiva pesquera andaluza en esas aguas y, sobre todo en una región asolada por el paro como es Andalucía, no es menos relevante el dato referente al empleo. La flota pesquera andaluza que faena en aguas marroquíes tiene directamente embarcados en sus buques a 6.601 tripulantes, el $27,5 \%$ del empleo pesquero directo de todo el sector.

Con la estadística actual es prácticamente imposible determinar con precisión el volumen de producción que se obtiene en el caladero; sin embargo, nosotros hemos querido -aún a riesgo de cometer error - dar una cifra orientativa de la misma y de su valor en primera venta. El dato, insistimos en que sea tomado sólo como indicativo, lo hemos calculado mediante un muestreo de la producción de diferentes unidades, en distintas épocas del año, dedicados a diversas especies y pertenecientes a puertos distintos. El total de la muestra alcanza al $10 \%$ de la flota pesquera andaluza que faena en Marruecos y los resultados obtenidos son que el montante de la producción total alcanza las $70.000 \mathrm{Tn} / \mathrm{año}$ (el $35 \%$ de toda la producción pesquera andaluza) y el valor de las capturas en primera venta se sitúa en torno a los 31.000 millones de pesetas (el 56,76\% del valor de toda la producción pesquera andaluza). Y ello, para una producción que se diversifica por grupos de especies según los porcentajes siguientes: $47 \%$ de marisco, $30 \%$ de sardinales, $10 \%$ de merlúcidos y un $13 \%$ de otras variadas especies.

\subsubsection{Costa portuguesa.}

Los bancos pesqueros de la costa portuguesa frecuentados por la flota andaluza van desde la desembocadura del Guadiana (Ayamonte-Vila Real de Sto. Antonio) hasta Cabo Sines, y sus actividades se circunscriben, por tanto, prácticamente al Algarve portugués.

Son caladeros muy ricos en crustáceos (cigala), y buenos en sardinales y moluscos. Son rentables para las flotas pesqueras andaluzas asentadas en el litoral onubense (Ayamonte, Isla Cristina y Huelva), dada la proximidad geográfica (que contribuye a disminuir los costes de explotación respecto a otros bancos pesqueros más lejanos) y la experiencia de nuestro marineros en el tipo de pesquerías que en ellos se realizan.

La actividad pesquera en el banco portugués la realizan dos tipos de flota: altura y litoral. La primera se dedica al arrastre, con buques de unas $130 \mathrm{Tn}$. de TRB y unos 300 HP en sus motores, y al cerco, con embarcaciones de unas $80 \mathrm{Tn}$. y motores similares a los anteriores, siendo sus especies de dedicación los crustáceos y los sardinales. Las segundas se dedican a las especies propias de las artes menores con que ejercitan su actividad y no tienen un TRB superior a las 20 Tn. ni motores con más de $60 \mathrm{HP}$. 
La flota que pesca en estas aguas está compuesta por 149 embarcaciones (el $6 \%$ del total andaluz) y llevan 1.134 hombres a bordo, lo que significa escasamente el $5 \%$ del total del empleo de la flota pesquera andaluza.

Su significación, como ya indicábamos en la localización de sus puertos base, viene dada por lo que representa para pueblos enteros (Isla Cristina y Ayamonte son los casos más relevantes) del litoral de la provincia de Huelva, cuyas flotas dependen exclusivamente de ese caladero, más que por su representación en el conjunto de la economía pesquera andaluza.

La relación de buques, con los datos del último acuerdo, es la que a continuación se detalla por tipos de pesca:

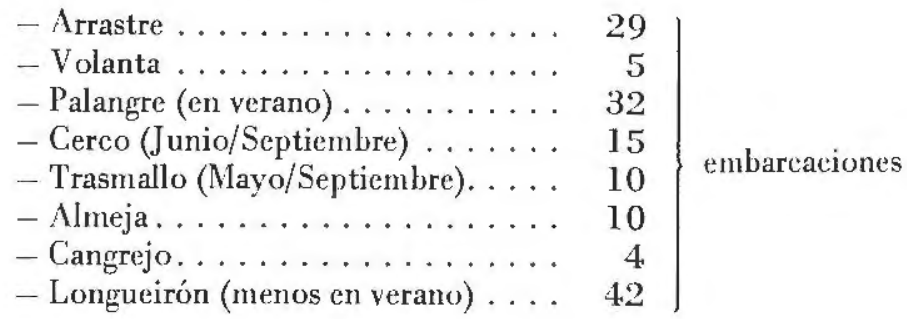

Es de tener en consideración que estas cifras resultan infladas respecto a la realidad, puesto que, por ejemplo, de arrastre apenas si se hace uso de veinticinco licencias.

\subsubsection{Otros bancos pesqueros.}

Estos son los propios de la flota pesquera de gran altura, que tiene su dedicación principal al marisco congelado; subsector congelador español que, como todos Vds. saben, tiene su sede en Huelva.

La flota está compuesta por 159 buques que faenan con arreglo a la siguiente distribución por caladeros:

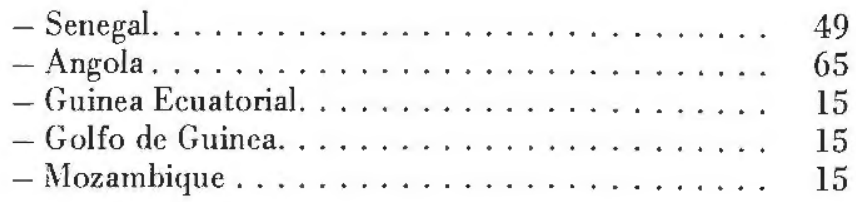

Son grandes unidades con un alto nivel tecnológico, desplazan una media de $400 \mathrm{Tn}$./buque y tienen una potencia media de $1.000 \mathrm{HP}$ en sus motores. Su edad media no sobrepasa los diez años y dan empleo a 2.720 hombres (el $12 \%$ del empleo pesquero andaluz). 
En la actualidad, estos caladeros son rentables para la flota pesquera an. daluza dedicada a esta actividad. Su producción media, según datos de la propia Asociación de Armadores, está por encima de las 60.000 Tn. anuales lo que viene a suponer un valor total de la producción en primera venta supe. rior a los doce mil millones de pesetas.

\subsection{Resumen histórico de los acuerdos de pesca.}

De forma breve, para situarnos ante el tema y sin entrar en mayores consideraciones, haremos un repaso de la trayectoria de los acuerdos cor terceros países, en materia de pesca, que afectan al sector pesquero andaluz.

\subsubsection{Con Marruecos.}

En 1969, se firma el Tratado de Fez que abre grandes expectativas a la flot pesquera andaluza y que posibilita el desarrollo de la misma en función de la nc existencia de limitaciones a su actividad.

Dos años despućs, en 1971, el Gobierno Marroquí denuncia el acuerdo y manifiesta su propósito de romper cl Tratado. Pese a ello, el sector pesquerc andaluz continúa faenando en el caladero sin especiales dificultades, aunque hace su aparición el fenómeno de los apresamientos.

En 1975, se mantiene el funcionamiento irregular de la flota a pesar de que Marruecos había decidido extender sus aguas jurisdiccionales a 70 millas er Enero de 1973 y arreciando en su labor apresora de nuestros barcos. En este año comienzan las negociaciones para el establecimiento de un nuevo acuerdc pesquero.

El cambio político que empieza a operarse en nuestro país, la confusa polí tica exterior de esos años - entre la que destaca los aeuerdos tripartitos de Ma drid, de cuyo contenido parece ser que nunca tendremos un conocimients exacto- y la falta de claridad en la ejecución de las tareas de vigilancia en la limitaciones de pesea impuestas por la propia Administración alauita, hacen qur los apresamientos empiecen a verse como algo cotidiano con lo que cada españo se encuentra al abrir la prensa del día.

En 1977, el recién constituido Parlamento español, ratifica (con los votos er contra de socialistas y comunistas) el acuerdo suscrito por la Administración es pañola con la marroquí. Seis años después, el Parlamento marroquí no había ra tificado el acuerdo.

Durante estos años se ha venido faenando en base a convenios de corta dura ción (un año máximo) cuya prórroga ha sido a base de elevados incrementos er los costes de explotación, entre los que no es el menor el amarre temporal de li flota, como ocurrió entre Febrero y Abril de 1980.

En definitiva, y tal como apuntaba el profesor Oya "...el conflicto pesquerc hispano-marroquí, no es sino una parte del contencioso que nuestro país tien con el Gobierno de Rabat...". Efectivamente, Marruecos utiliza la necesidad qu tiene la flota andaluza de pescar en sus aguas como baza política en eualquier. 
de los contenciosos que nos enfrentan -Ceuta, Melilla, reconocimiento de la RASD..., como fórmula de presión económica- solicitud de paso sin arancel de sus agrios camino de los mercados europeos, como instrumento de desviación de sus propios problemas internos -véase ahora con la fuerte crisis de gobierno que padece- dándole gran publicidad al enfrentamiento pesquero con España... e incluso como baza política internacional, al adoptar actitudes que más parecen favorecer a otras potencias económicas que a su propio interés, como veremos más adelante.

Las dificultades principales -en el orden estricto de las pesquerías en sus aguas - de los acuerdos bilaterales con Marruecos, se han centrado en:

- Exigencia de reducción del esfucrzo pesquero en sus aguas jurisdiccionales y siempre fuera de las doce millas.

- Permanentes elevaciones de los cánones.

- Exigencias en concesiones de créditos oficiales interestatales a bajo interés y larga duración.

- La sustitución de mano de obra andaluza por marroquí en las embarcaciones.

- Las ventanas de seguridad que impone el gobierno de Rabat.

- Y el más grave, a nuestro entender, la inseguridad de futuro para la actividad con prórrogas no superiores al año y que en los últimos años hemos observado como casi grotescas con la prórroga por un mes (Julio) primero y por días después.

La firma del último acuerdo - Agosto 1983- ha venido a salvar de forma clara ésta postrera dificultad y en ello radica el principal éxito del mismo; en su duración de cuatro años. Para ello, no cabe duda, ha habido que hacer grandes concesiones en el resto de los apartados antes citados. Así, el esfuerzo pesquero se verá reducido en un $40 \%$ al final del período, los incrementos de los cánones serán del $70 \% \mathrm{y}$, en fin, se le concede al Reino de Marruecos un crédito de 150 millones de dólares a un interés del 4 \% y con ocho años de carencia.

\subsubsection{Con Portugal.}

También en este caso el primer convenio data de 1969, coucretamente del 13 de Diciembre, y en él lo más destacable es: su duración (20 años), las zonas afectadas (6 a 12 millas) y el mantenimiento del derecho consuctudinario entre los pescadores de ambos países, para el establecimiento de los criterios reguladores de la actividad en las zona marítimas fronterizas.

A partir del derrocamiento de la dictadura salazarista, el sector pesquero portugués presiona a su gobierno para que denuncie el acuerdo por considerarlo claramente lesivo para sus intereses y haber surgido de un pacto entre dictadores. Comienzan así las reivindicaciones lusitanas sobre el establecimiento de plazos para la supresión de la pesca de arrastre y cerco dentro de las doee millas. De tal forma que se habla de suprimir el arrastre entre las seis y las doce millas para 1982 y el cerco para 1985. 
En Abril de 1977, Portugal hace uso de sus derechos sobre las doscienta millas de su plataforma contineutal narítima y la declara Zona Económic. Territorial (ZET). Esta decisión de la administración portuguesa conlleva l. necesidad de una nueva negociación para la zona comprendida entre las doce : las doscientas millas, sin limitación en el acuerdo de 1969.

Así las cosas, en Marzo de 1979 se reducen las licencias de arrastre a 50, d. las cuáles sólo 25 son para faenar entre las 6 y 12 millas. Se establece el sistem de ventanas (o zonas protegidas), se ponen limitaciones - en principio muy rígi das después más flexibles- al porcentaje de capturas de crustáceos y, en conse cuencia, pierde sentido el acuerdo de Juntas Fronterizas.

Este acuerdo se prorroga hasta Febrero de 1981 y, posteriormente, cuatrı meses más con una reducción del $35 \%$ en las lieencias que dió origen a uı amarre voluntario de la flota pesquera que faenaba en esas aguas como protesta.

Por fin, los días 21 y 22 de Septiembre de 1981, en Madrid, se firma el pro tocolo adicional a los acuerdos de 1969 y 22 de Septiembre de 1978 por cl qu. se reglamentaban los planes de pesca en el caladero portugués desde el primerı de Octubre hasta la entrada de España y Portugal en la C.E.E.

Con este acuerdo se conseguían tres cuestiones importantes: incrementa las licencias de arrastre y palangre, eliminar las ventanas y la supresión de la limitaciones en el tonelaje de capturas de crustáceos.

Así se llegó a finales de 1982 en que fue imposible cumplimentar un nue vo acuerdo y como consecuencia de ello, dada la denuncia portuguesa del an terior, la flota pesquera andaluza que faenaba en el caladero portugués per manece amarrada desde Enero del presente año, como de todos es sabido.

Las principales dificultades surgen de la consideración de los crustáceo (cigala), por parte portuguesa, como especie sedentaria y su negación a qu el sector pesquero andaluz lleve a cabo este tipo de actividad en sus aguas e] el futuro. Por medio están unos derechos históricos que protege el Tribuna de la Haya y a los que la parte española no está dispuesta a renunciar.

\subsubsection{Con otros países (Senegal, Angola, Mozambique...).}

Cono ya decíamos al hablar de la significación económica de los mismos, e ellos faena la flota de gran altura andaluza y en nn principio los acnerdos surgif ron de la iniciativa privada que incluso llegó a establecer contratos con algunc de estos países, arrastrando después al Gobierno a formalizar o "legalizar" lc acuerdos con el refrendo del Estado y en el marco de acuerdos bilaterales entr países soberanos.

Cada día son más difíciles las negociaciones para establecer acuerdos con lc gobieruos de los países donde se encuentrau estos caladeros. De una parte, pol que los países del Tercer Mundo han visto en sus recursos pesqueros una gra fuente de ingresos y elevan continuamente las contraprestaciones económica: De otra, por la inestabilidad política de la mayoría de sus gobiernos de estc países que hacen inseguro el cumplimien to futuro de los acuerdos. 
Los acuerdos suelen ser anuales y, en ocasiones, bianuales pero, las dificultades apuntadas hacen necesaria la búsqueda de nuevos ealaderos alternativos para que ante cualquier eventualidad que impida el desarrollo normal de la aetividad ésta pueda tener continuidad en otras zonas.

\section{PERSPECTIVAS ANTE LA INTEGRACION DE ESPAÑA EN LA C.E.E. Y SUS REPERCUSIONES EN LA ECONOMIA PESQUERA ANDALUZA.}

Dado que los barcos de pesca andaluces no faenan en aguas comunitarias, el cambio de perspectivas que podría suponer la integración de España en la Comunidad Económica Europea se observa desde el sector pesquero andaluz con excepticismo no exento de ciertas dudas.

Se piensa, no sin cierta lógica, que la integración en la Comunidad afectará principalmente a las flotas gallega y cantábrica; y que los beneficios -en el caso de que los hubiere- para las pesquerías andaluzas serían escasos y difíciles de determinar "a priori".

Hablar de las perspectivas de los acuerdos con terceros países - países, no lo olvidemos, muchos de ellos con una inestabilidad política manifiesta e insertos en un marco internacional de tensiones permanentes- es hacer un poco de futurología, con el riesgo de que los factores exógenos al estricto análisis pesquero $\mathrm{y}$, por tanto, difícilmente cuantificables den al traste con muchas de las ideas que hoy nos pueden aparecer como meridianamente claras.

Una cuestión es evidente, en el momento de nuestra integración los acuerdos establecidos por Madrid serían denunciados y Bruselas tomaría la dirección de cara a establecer acuerdos. $\mathrm{Y}$ así, es un hecho constatable que en la actualidad, cuando se habla de nuevos acuerdos nuestros interlocutores empiezan a plantear la fórmula de "hasta el ingreso de España en la C.E.E." como límite de duración máximo, en un intento de no aparecer hipotecados sus derechos ante unas nuevas e inevitables negociaciones en un marco más amplio.

Aún a costa de todas las incertidumbres reseñadas, pensamos que debemos intentar el análisis y en ese sentido lo haremos primero con carácter general en torno a lo positivo y negativo que para la Economía Pesquera Andaluza puede tener la integración, para pasar después a un análisis más detallado según las distintas zonas de faenaje de la flota pesquera de nuestra región.

\subsection{Análisis General.}

Couno todo cambio de marco en las relaciones económicas, entendemos que la integración de España en el Mercado Común presenta una serie de aspectos positivos y otros no tanto, para la economía pesquera andaluza. 
Entre los primeros, y principalmente, existe la confianza de que el cam. bio de centro negociador de Madrid a Bruselas, posibilitaría una mayor fues za en la negociación con terceros países en orden a la consecución de acues dos más duraderos, estables y ventajosos que los actuales.

De una parte, los "puntos negros" (exigencias de tipo reivindicativo pol] tico) de algunas de las negociaciones actuales no tendrían razón de ser; y d otra, las compensaciones económicas y técnicas que puede ofrecer la C.E.E son mayores que las que podemos ofrecer nosotros.

En el ámbito de la comercialización, la adaptación a las nuevas condicic nes del mercado mejoraría sustancialmente los actuales canales de comercial zación haciéndolos más transparentes. Acercar los productos a los circuito comerciales potenciando un mayor peso específico de los mismos en la detej minación de los precios en primera venta, la mejora en la presentación de producto, el mayor rigor en la calidad y en las condiciones higiénico-sanite rias del mismo... son todas cuestiones que a pesar de la rigidez de principio aceptarlas sólo pueden venir a mejorar la rentabilidad de la actividad.

$\mathrm{Y}$, en fin, en este mismo sentido la expansión del mercado, puede benef ciar a determinados productos andaluces. Tal es el caso de los mariscos cor gelados y las conservas de pescado. Los primeros una vez solucionado el tem del ácido bórico, y dado que no cuentan con competidores a nivel europec Los segundos, porque verían mejorada la posición de sus productos en $€$ mercado europeo al no verse estrangulada su comercialización por las tasa arancelarias que padece en la actualidad.

De los aspectos negativos, el tema más preocupante es el de la reestruı turación de la flota.

La C.E.E. manifiesta su preocupación por nuestro montante de flota, co lo que exige una reducción de la flota pesquera y, consecuentemente, de ] andaluza. En este contexto, la "Europa Azul" pretende que España reduzc su potencial pesquero antes de su entrada como miembro de pleno derech en la Comunidad, con lo que evitaría el hacerse cargo de los costes de la ree tructuración que le corresponderían en caso de que se llevase a cabo co posterioridad a nuestro ingreso.

Llevar a cabo la reestructuración -o reconversión- de la flota pesquer andaluza parece no sólo inevitable sino necesario. Pero, hacerlo a corto pla zo supondría un coste social para la economía pesquera andaluza, en partic lar, y para el conjunto de la economía regional, en general, difícil de afronta en la coyuntura actual.

Me van a permitir que en este punto haga una serie de consideracion que en Andalucía nos llaman poderosamente la atención.

En las últimas y laboriosas (por sus dificultades y duración) negociacic nes con Marruecos, este país se mostró inflexible en la reducción del esfuerz pesquero, llegando en algunos momentos a pedir una reducción del 70 \% del mismo en un año. Esto unido a la importancia que para sus ingresos tiı 
nen los pagos del sector pesquero andaluz por faenar en sus aguas y la merma que ello supondría para las arcas alauitas, hace que manifestemos nuestra extrañeza y nos preguntemos ¿porqué ese empeño marroquí?.

Un inciso para una anécdota. En plena avidez mundial de dólares el Gobierno de Marruecos estaba dispuesto a cobrar los cánones en unidades monetarias de la comunidad (e.c.u.).

Las mismas insistencias en reducción del número de barcos faenando en sus caladeros, con otros muchos factores concomitantes, nos exige Portugal... o Senegal, por poner otro ejemplo.

Parece haber una coincidencia - que no creemos casual-, con padecimiento en el sector pesquero andaluz de sus consecuencias, entre las exigencias de la C.E.E. y las de terceros países no miembros (principalmente, los de influencia francófona) para que nuestra flota pesquera se reduzca en el más breve espacio de tiempo por imposibilidad de faenar.

Otro aspecto que se observa como negativo es el del mercado de productos pesqueros. Somos, y ya se ha dicho repetidamente, el mayor cosumidor de pescado de Europa. Y avalando este dato, desde Andalucía podemos decir que Cádiz, Huelva y Málaga tienen el mayor índice de consumo de proteínas de origen marino "per cápita" de todo el continente. Lógicamente, estas cifras no se le escapan a la Comunidad que nos ve, pese a sus quejas sobre nuestra estructura productiva, claramente como consumidores.

Las repercusiones que pueden tener en los precios del mercado interior el libre acceso al mismo de los productos pesqueros comunitarios es muy preocupante en Andalucía. Máxime cuando ya en la actualidad se observa lo influenciables a la baja que aquellos son por las importaciones actuales. Ejemplo claro de cuanto relatamos es la caída en los precios que suponen las entradas masivas de chirla italiana, que está colocando al correspondiente subsector onubense en riesgo de desaparecer como actividad.

Y esta idea está intimamente ligada a la anterior. Efectivamente, se piensa que el romper nuestra capacidad de autoabastecimiento y generar una demanda insatisfecha, a través de una drástica reducción de la flota ampliaría el mercado para el resto de los países comunitarios y posibilitaría, incluso, el cambio de gustos en el consumo (idea a la que no son ajenas las industrias cárnicas europeas).

Por último es de constatar la posibilidad de irrupción de las multinacionales de la agroalimentación en Andalucía. Dado el peso específico de la producción del sector primario andaluz (pesca incluído) no es descabellado preveer una entrada de aluvión de empresas multinacionales dedicadas a la actividad agroalimentaria. De hecho, ya existen hoy en nuestra tierra empresas de este tipo para productos agrícolas congelados y transformados (pastas), con una perfecta red de comercialización y que de cara a su expansión el sector más consecuentemente interesante para aprovechar las economías externas que generan sus actividades actuales, sería - sin duda- el sector pesquero. 


\subsection{Análisis de las repercusiones de la integración de España en la C.E.E. para la Economía Pesquera Andaluza por zonas de faenaje.}

\subsubsection{El caso de la costa sahariano-marroquí.}

Parece ser que en el momento de la integración no habrá ningún acuerdc en vigor, puesto que la Administración Marroquí tiene la intención de que así sea y las firmas de futuros acuerdos tendrán como fecha tope el instante de nuestra entrada en la C.E.E.

Es el caladero donde se espera de forma más ilusionada que la negocia. ción desde Bruselas imposibilite la utilización de las reivindicaciones políticas como factor determinante en el marco de los acuerdos pesqueros.

Las dificultades comerciales que, según los marroquíes, España pone a paso de sus productos hacia Europa perderían peso como argumento en las negociaciones pesqueras, al inscribirse en el marco de las relaciones del blo. que capitalista europeo con un país fuertemente dependiente del mismo.

Aunque se obseva que no existe, en la actualidad ninguna flota comunita. ria interesada en faenar en aguas del banco sahariano-marroquí (en todo casc alguna portuguesa, y sin gran relevancia, y lo haría siempre en el marco de una integración conjunta en la C.E.E.) porque ni el tipo de especies ni su cali. dad son hoy objeto de-interés para las pesquerías comunitarias, entendemos que este no debe ser un argumento definitivamente favorable de cara a un futura repartición de cuotas de pesca. Y no lo debe ser dado que el avance tecnológico en materia pesquera, unido a la búsqueda de nuevos caladeros por parte de las empresas pesqueras de todo el mundo, en la actualidad, pue. den hacer variar en el futuro esta idea.

A este respecto, la duda se ve aumentada si, como exponíamos en nues. tro análisis general, existe la posibilidad de que uniendo los bajos costes de explotación que propicia la proximidad del caladero y la facilidad a un libre acceso al desembarco de la producción en nuestros puertos y, consecuentemente, a nuestro mercado, algunas empresas comunitarias vean una salida a sus escasos rendimientos en otras aguas en el hecho de faenar en la costa sahariano-marroquí, entrando en competencia con nuestra flota pesquera.

Habría entonces que tener prevista esta situación. Pues, y a pesar de que quizás sea rizar el rizo de las elucubraciones más osadas, no parece arriesgadc pensar que si alguna flota comunitaria tuviese esa intención las fórmulas po. drían ir desde la constitución de empresas mixtas con entidades españolas (no necesariamente andaluzas) o bien como flotas abastecedoras de materia prima a las industrias transformadoras multinacionales ya referidas.

Ambas posibilidades, como todo el análisis que estamos haciendo, tienen lecturas negativas como las apuntadas y lecturas positivas. Entre éstas una es la constatación de que Andalucía posee el capital humano con conocimientc 
perfecto del caladero y su puesta en práctica significaría la absorción de una mano de obra que, con una reestructuración por medio, sería excedentaria en el sector pesquero andaluz.

En otro sentido, el hecho de un acuerdo Bruselas-Rabat ofrecería un marco de responsabilidad en el cumplimiento del mismo y obligaría a un mayor rigor en el desarrollo de la actividad por parte del sector pesquero andaluz y a una mayor seriedad en las tareas de vigilancia marroquí que acabe con la lacra de los apresamientos y todo el fenómeno de ocultismo social que hay en el transfondo de los mismos en los últimos diez años, dotando a la pesca de los parámetros normales de una actividad económica regular ejercida en otros países y quitándole el aspecto de "película de piratas" con que, por ambas partes, se reviste en algunas ocasiones.

\subsubsection{El caladero portugués.}

De cara a la integración de España en la C.E.E., dos son las características que diferencian a este caladero de los otros donde ejercita su actividad la flota pesquera andaluza. Una primera, la que viene dada por el hecho de que Portugal está llamada a integrarse en la Comunidad casi al unísono que España; la segunda, no menos importante, es la necesidad que tiene la flota pesquera del Algarve portugués de faenar en aguas del litoral andaluz.

Se espera, por tanto, que la integración de ambos países desbloquee definitivamente situaciones como las que se vienen padeciendo en los últimos años, permitiéndole al sector pesquero andaluz faenar libremente entre las doce y las doscientas millas en virtud de la Política Pesquera Comunitaria (P.P.C.) acordada en el presente año, con las cuotas de pesca que se le impongan y que no parecen difíciles de asumir salvo en el caso de los barcos del longueirón que es un subsector a ordenar dentro del propio sector pesquero andaluz por ser una actividad claramente depredadora e impresentable a ningún acuerdo internacional en su actual configuración.

El nudo gordiano de las discrepancias se centra en el acuerdo de 1969 y los derechos históricos que el mismo consagra. Y es, curiosamente, en este punto donde la P.P.C. en lugar de aclarar ha contribuido a generar más dudas respecto a la posible renuncia de España a los mismos, pues, en ella se sienta el precedente establecido entre Francia y el Reino Unido -en el marco de la Comunidad- por el cual algunas flotas francesas podrán faenar entre las seis $\mathrm{y}$ las doce millas en aguas inglesas.

Lógicamente, este hecho invita a pensar que una actitud firme en la actualidad respecto al mantenimiento de los derechos históricos de nuestra flota pesquera, puede tener unas muy importantes consecuencias en el futuro y una vez que ambos países sean miembros del Mercado Común Europeo. 
Ante esta situación nosotros entendemos que dado que nuestro es el mer. cado, la tecnología y el factor humano capaz de explotar con buenos rendi. mientos los recursos del caladero y que de Portugal son las riquezas de su li. toral, la salida más positiva y acertada de cara a la integración conjunta seríe el acceder a la misma con empresas mixtas lusitano-andaluzas para la explota ción de los caladeros. Salvando con ello la divergencia en tomo a los arrastre ros y facilitando la normal actividad de las flotas de cerco a uno y otro ladc de la frontera del Guadiana que interesa a ambas comunidades pesqueras.

\subsubsection{En los caladeros lejanos.}

El alto nivel tecnológico de las unidades de la flota pesquera de gran altu ra, su infraestructura y funcionamiento empresarial claramente diferenciadc del resto de las flotas, la apertura de mercado para sus productos - tal y co mo exponíamos en el análisis general- con la consiguiente consolidación d Huelva como el mayor puerto descargador de marisco congelado de Europe y el hecho de ser un subsector pesquero con un buen nivel de rendimientos hacen que no aparezcan dudas sobre lo positivo de la integración en la Comu nidad, y principalmente por cuatro poderosas razones:

- Dotación de transparencia en el acceso de sus productos al mercado y er la formación de los precios, al tener que circunscribirse a las normas co munitarias.

- Apoyo investigador, del que está necesitado el sector, para la búsqueda J acceso a caladeros alternativos.

- Facilidad de acceso a una tecnología en constante cambio.

- Seguridad y estabilidad en los acuerdos con terceros países por la mayo: presión negociadora que sobre los mismos pueda ejercer la Comunidad.

Por último, y aún dando todos por hecha nuestra integración en el Mer cado Común Europeo, creemos que como contraste de nuestro análisis seríc interesante hacer la reflexión contraria a la que nos venimos haciendo; es de cir, ¿qué pasaría si no se produce la integración en la Comunidad?.

Esta pregunta cambia, de manera sustancial, el planteamiento de las pers pectivas futuras. Habría, en este supuesto, que hacer el análisis partiendo de nuestras propias fuerzas y entonces, conscientes de la voluntad política de le actual administración pesquera, nos preguntaríamos iseremos capaces de or denar y redimensionar consecuentemente el sector pesquero andaluz a medic plazo?.

La clave está en el factor tiempo. Con un sector de litoral perfectamente delimitado y ordenado, con los caladeros propios evaluados y estimada si potencialidad de esfuerzo pesquero y con una estrecha vigilancia sobre le pesca y comercialización de inmaduros, el sector pesquero andaluz puede $\mathrm{J}$ 
debe ser reordenado de tal forma que la dependencia de la economía pesquera andaluza de caladeros foráneos sea mucho menor que la actual. Y ello, a medio plazo, es perfectamente posible y alcanzable.

Una mayor participación de los productores, a través de fórmulas asociativas de los mismos en el marco de unas Cofradías de Pescadores cumplidoras del verdadero papel que están llamadas a desempeñar, dotaría a los canales de comercialización de una mayor transparencia y ajustaría los precios en primera venta a las necesidades de rentabilidad de la flota.

Con todo, un descenso en la dependencia de las pesquerías andaluzas fuera de las aguas que le son propias, colocaría al sector en una posición más ventajosa de cara a las negociaciones con otros países y, dado que estos necesitan los ingresos que les proporciona el sector pesquero andaluz, haría más factible la consecución de acuerdos estables y duraderos.

Asímismo, con el carácter conexo que las actividades tienen, en el mercado de una reestructuración del sector y viéndola desde una perspectiva integral, sería necesaria la potenciación de nuestra propia industria de transformación en aras a no dejar en manos de las multinacionales este campo.

En resumen, si con la entrada en la C.E.E. los cambios estructurales hay que efectuarlos con tal premura de tiempo que el coste social para Andalucía se volviese insoportable $y$, de otra parte, las ventajas derivadas de la integración no parecen tan nítidas como para afrontar aquel, entendemos que más vale que ordenemos nuestra casa sin prisas... pero, sin pausas, claro está. $\mathrm{Y}$, en todo caso, si ello es factible hacerlo en el marco de la C.E.E. mejor que mejor. 